\title{
Pismis 20: One or two clusters?^
}

\author{
A. M. Orsatti ${ }^{1,2, \star \star}$, E. I. Vega ${ }^{1,3, \star \star}$, and H. G. Marraco ${ }^{1, \star \star}$ \\ 1 Facultad de Ciencias Astronómicas y Geofísicas, Universidad Nacional de La Plata, Paseo del Bosque s/n, \\ 1900 La Plata, Argentina \\ 2 Instituto de Astrofísica de La Plata, (IALP, CONICET), Paseo del Bosque s/n, 1900 La Plata, Argentina \\ ${ }^{3}$ Instituto de Astronomía y Física del Espacio, C. C. 67 Suc. 28, 1428 Buenos Aires, Argentina
}

Received 30 December 2002 / Accepted 16 May 2003

\begin{abstract}
We present $U B V R I$ polarimetric observations of stars in the direction of the open cluster Pismis 20 and WR 67. It is found that the observed members segregate polarimetrically into two groups, with different associated mean $P_{\max }(4.31$ and $6.08 \%$ respectively). Although in the literature these stars are considered as members of the same cluster, the polarized light from these groups clearly differs. There are two possible explanations: very efficient polarizing dust particles exists between certain members of the cluster; or else Pismis 20 is composed of different groupings superimposed along the line of sight. WR 67 belongs to the group more affected by the dust.
\end{abstract}

Key words. ISM: dust, extinction - open clusters and associations: individual: Pis20 - stars: individual: WR67 - technique: polarimetric

\section{Introduction}

Pismis 20 (C1511-588) is a very young and concentrated group of stars, $2^{\prime}$ in diameter, located at $l=320.51$ and $b=-1.20$ and lying in the Norma-Scutum (-II) arm of the Galaxy. It was identified as a group by Pismis (1959) and the first photometric and spectroscopic observations were made by Lyngå (1968; hereinafter LY68), who found for this cluster an age of $5 \times$ $10^{6} \mathrm{yr}$, with a mean excess $\overline{E_{B-V}}=1.22 \mathrm{mag}$. Most of the absorption is assumed to be produced in the Local and the Sagittarius-Carina arms. The cluster has been investigated by Paunzen et al. (2002) in a search for chemically peculiar stars, with negative results. Nearly $2^{\prime}$ from the central core of the cluster is WR 67 (LSS 3329, HD 134877), a WN6 + OB? Wolf-Rayet star (van der Hucht 2001) whose membership of Pismis 20 has been in doubt for several years, even though some investigations suggest it (LY68, Lundström \& Stenholm 1984; Vázquez et al. 1995, hereinafter VZ95; Massey et al. 2001).

The distance to this open cluster has been a matter of controversy through the years. In the literature it runs widely between the extreme values $4.4 \mathrm{kpc}$ (LY68, Papadópulos 1985) and $2.55 \mathrm{kpc}$ (Peterson \& FitzGerald 1988), as well as $2.07 \mathrm{kpc}$

Send offprint requests to: $\mathrm{H}$. G. Marraco, e-mail: hmarraco@fcaglp.unlp.edu.ar

* Based on observations obtained at Complejo Astronómico El Leoncito, operated under agreement between the Consejo Nacional de Investigaciones Científicas y Técnicas de la República Argentina and the Universities of La Plata, Córdoba, and San Juan.

$\star \star$ Member of the Carrera del Investigador Científico of CONICET. as found by Moffat \& Vogt (1973) which took in consideration only the most luminous stars of the group. In between those extremes there exist other determinations: $3.98 \mathrm{kpc}$ (Lortet et al. 1987), $3.6 \mathrm{kpc}$ (VZ95), and $3.27 \mathrm{kpc}$ (Turner 1996). Sources of errors are the heavy reddening that hampers the study of the faint cluster main sequence, photometric errors, peculiarities, binarity, etc.

This investigation analyzes the polarization in the light from stars in the direction of Pismis 20 with the aim of investigating the properties of the dust causing the polarization, and also to confirm with this technique the membership of WR 67 in the cluster.

\section{Observations}

We have selected for polarimetric observation 15 suspected members of Pismis 20 (stars Nos. 1-3, 7-13 and 1a (WR 67) according to LY68; and stars Vz121, Vz128, Vz 148 and $\mathrm{Vz} 182$ (identifications from VZ95 ). We have also observed 5 non-members (Nos. 4 and Vz 14, Vz 30, Vz 107 and Vz 183) recognized as such in the cited investigations.

Observations in the $U B V(R I)_{\mathrm{KC}}$ bands (KC: Kron-Cousins, $\lambda_{U_{\mathrm{eff}}}=0.36 \mu \mathrm{m}, F W H M=0.05 \mu \mathrm{m} ; \lambda_{B_{\mathrm{eff}}}=0.44 \mu \mathrm{m}$, $F W H M=0.06 \mu \mathrm{m} ; \lambda_{V_{\text {eff }}}=0.53 \mu \mathrm{m}, F W H M=$ $0.06 \mu \mathrm{m} ; \lambda_{R_{\mathrm{eff}}}=0.69 \mu \mathrm{m}, F W H M=0.18 \mu \mathrm{m} ; \lambda_{I_{\mathrm{eff}}}=0.83 \mu \mathrm{m}$, $F W H M=0.15 \mu \mathrm{m}$ ) were carried out using the five-channel photopolarimeter of the Torino Astronomical Observatory attached to the $2.15 \mathrm{~m}$ telescope at the Complejo Astronómico El Leoncito (San Juan, Argentina). They were performed on 3 nights (March 20-22) in 1998 and 2 nights (March 13-14) in 
1999. No systematic differences were found between observing runs. Standard stars for null polarization and for the zero point of the polarization position angle were taken from Clocchiati $\&$ Marraco (1988). Table 1 lists the observed stars in the direction of Pismis 20, the percentage polarization $\left(P_{\lambda}\right)$, the position angle of the electric vector $\left(\theta_{\lambda}\right)$ in the equatorial coordinate system and their respective mean errors for each filter. We indicate also the number of $60 \mathrm{~s}$ independent integrations with each filter. Star identifications are taken from LY68 and VZ95, and non-members according to any of the investigations are indicated in this table and also in Table 2 below.

\section{Results}

By observing the amount of interstellar polarization in several bandpasses, the wavelength at which maximum polarization $\left(P_{\max }\right)$ occurs can be computed. This wavelength $\lambda_{\max }$ is a function of the optical properties and characteristic particle size distribution of the aligned grains (McMillan 1978; Wilking et al. 1980). The maximum polarization $P_{\max }$ has been calculated by fitting the observed polarization in the $U B V R I$ bandpasses to the standard Serkowski's polarization law,

$$
P_{\lambda} / P_{\max }=\exp \left[\begin{array}{lll}
-k & \ln ^{2}\left(\lambda_{\max } / \lambda\right)
\end{array}\right]
$$

and adopting the parameter $k=1.15$ (Serkowski 1973). If the polarization is well represented by this relation (meaning that the measured polarization is of interstellar origin), $\sigma_{1}$ (the unit weight error of the fit) should not be higher than 1.5 , because of the weighting scheme; a higher value could indicate the presence of intrinsic polarization.

Table 2 lists the $P_{\max }, \lambda_{\max }$ and $\sigma_{1}$ values for the observed stars. We also list in the last column spectral types obtained from the literature. The mathematical expression used to determine the individual $\sigma_{1}$ values can be found there as a footnote.

\section{Analysis and discussion}

From their measures, 7 objects (stars 1, 2, 10, 13, Vz107, Vz148, WR 67) have a value for the unit weight error of the fit $\left(\sigma_{1}\right)$ above 1.5. Such a value may be considered as a limit due to the weighting scheme, that is, a higher value could be indicative of the presence of intrinsic polarization in the light from a star. Numero 7 (with a $\sigma_{1}$ value of 1.45 ) is also a possible member of this group. Numeros 4 and 12 have their fitted $\lambda_{\max }$ shorter than the average general value for the interstellar medium $(0.55 \mu \mathrm{m}$; Serkowski et al. 1975), amounting to $0.48 \mu \mathrm{m}$ in both cases. This is another criterion which gives a clue to intrinsic polarization.

The most notable $P_{\lambda}$ and $\theta_{\lambda}$ vs. $\lambda$ plots for stars with indications of intrinsic polarization are shown in Fig. 1, where the solid curve denotes the standard Serkowski's curve (1), the law valid for an interstellar origin in the measured polarization. The presence of intrinsic polarization in the light from a star causes a mismatch between observations and the Serkowski's curve fit, and/or a rotation in the position angle of the polarization vector. The abovementioned mismatch is clearly seen in each plot; but the rotation in the position angle with $\lambda$ is not
Table 1. UBVRI polarimetric observations of Pismis 20.

\begin{tabular}{|c|c|c|c|c|c|c|c|}
\hline $\operatorname{Star}^{a}$ & Id. ${ }^{b}$ & Filter & $\begin{array}{l}P_{\lambda} \\
\%\end{array}$ & $\begin{array}{l}\epsilon_{\mathrm{p}} \\
\%\end{array}$ & $\begin{array}{c}\theta_{\lambda} \\
\circ\end{array}$ & $\begin{array}{c}\epsilon_{\theta} \\
\circ\end{array}$ & $n^{c}$ \\
\hline \multirow[t]{5}{*}{1} & Vz52 & $U$ & 7.14 & 1.13 & 60.3 & 4.5 & 8 \\
\hline & & $B$ & 6.79 & 0.97 & 61.3 & 4.0 & \\
\hline & & $V$ & 5.64 & 0.15 & 60.8 & 0.7 & \\
\hline & & $R$ & 6.02 & 0.20 & 62.1 & 1.0 & \\
\hline & & $I$ & 5.41 & 0.37 & 64.8 & 2.0 & \\
\hline \multirow[t]{5}{*}{2} & Vz72 & $U$ & 2.80 & 0.53 & 45.5 & 5.5 & 6 \\
\hline & & $B$ & 4.43 & 0.65 & 64.1 & 2.7 & \\
\hline & & $V$ & 5.99 & 0.30 & 61.6 & 1.4 & \\
\hline & & $R$ & 6.19 & 0.27 & 61.0 & 1.3 & \\
\hline & & $I$ & 4.53 & 0.53 & 61.3 & 3.3 & \\
\hline \multirow[t]{5}{*}{3} & Vz91 & $U$ & 3.99 & 2.32 & 15.3 & 5.6 & 4 \\
\hline & & $B$ & 4.28 & 2.05 & 10.9 & 1.3 & \\
\hline & & $V$ & 3.18 & 0.53 & 63.6 & 4.7 & \\
\hline & & $R$ & 2.97 & 0.93 & 58.0 & 8.7 & \\
\hline & & $I$ & 4.31 & 1.41 & 64.3 & 9.1 & \\
\hline \multirow[t]{5}{*}{$4 *$} & Vz77 & $U$ & 0.92 & 0.23 & 53.9 & 7.0 & 12 \\
\hline & & $B$ & 2.07 & 0.30 & 66.0 & 4.2 & \\
\hline & & $V$ & 1.60 & 0.25 & 64.9 & 4.5 & \\
\hline & & $R$ & 1.48 & 0.20 & 64.6 & 3.9 & \\
\hline & & $I$ & 1.46 & 0.32 & 58.2 & 6.1 & \\
\hline \multirow[t]{5}{*}{7} & Vz104 & $U$ & 2.42 & 0.47 & 62.9 & 5.5 & 6 \\
\hline & & $B$ & 4.49 & 0.42 & 65.4 & 2.7 & \\
\hline & & $V$ & 3.98 & 0.35 & 56.7 & 2.5 & \\
\hline & & $R$ & 3.84 & 0.35 & 62.8 & 2.6 & \\
\hline & & $I$ & 3.14 & 0.58 & 55.2 & 5.2 & \\
\hline \multirow[t]{5}{*}{8} & Vz123 & $U$ & 4.56 & 0.29 & 76.1 & 1.8 & 4 \\
\hline & & $B$ & 5.77 & 0.07 & 62.9 & 0.3 & \\
\hline & & $V$ & 5.79 & 0.22 & 61.5 & 1.1 & \\
\hline & & $R$ & 6.05 & 0.24 & 62.4 & 1.1 & \\
\hline & & $I$ & 5.14 & 0.30 & 61.3 & 1.7 & \\
\hline \multirow[t]{5}{*}{9} & Vz125 & $U$ & 3.90 & 0.28 & 71.0 & 2.0 & 4 \\
\hline & & $B$ & 4.84 & 0.45 & 64.7 & 2.6 & \\
\hline & & $V$ & 3.76 & 0.64 & 61.3 & 4.9 & \\
\hline & & $R$ & 4.92 & 0.51 & 64.5 & 2.9 & \\
\hline & & $I$ & 3.80 & 0.78 & 61.4 & 5.8 & \\
\hline \multirow[t]{5}{*}{10} & Vz126 & $U$ & 6.18 & 1.53 & 62.3 & 7.0 & 4 \\
\hline & & $B$ & 6.23 & 0.30 & 61.7 & 1.4 & \\
\hline & & $V$ & 6.14 & 0.34 & 62.6 & 1.6 & \\
\hline & & $R$ & 6.98 & 0.12 & 65.2 & 0.5 & \\
\hline & & $I$ & 5.59 & 0.22 & 64.8 & 1.1 & \\
\hline \multirow[t]{5}{*}{11} & Vz106 & $U$ & 5.87 & 1.46 & 70.5 & 7.0 & 4 \\
\hline & & $B$ & 6.11 & 0.30 & 62.8 & 1.4 & \\
\hline & & $V$ & 6.17 & 0.27 & 66.7 & 1.2 & \\
\hline & & $R$ & 6.25 & 0.17 & 65.8 & 0.8 & \\
\hline & & $I$ & 5.29 & 0.35 & 67.8 & 1.9 & \\
\hline
\end{tabular}

a common characteristic of the whole group of stars with $\sigma_{1}$ higher than 1.5. 
Table 1. continued.

\begin{tabular}{|c|c|c|c|c|c|c|c|}
\hline $\operatorname{Star}^{a}$ & Id. $^{b}$ & Filter & $\begin{array}{c}P_{\lambda} \\
\%\end{array}$ & $\begin{array}{l}\epsilon_{\mathrm{p}} \\
\%\end{array}$ & $\begin{array}{c}\theta_{\lambda} \\
\circ\end{array}$ & $\begin{array}{c}\epsilon_{\theta} \\
\circ\end{array}$ & $n^{c}$ \\
\hline 12 & Vz75 & $\begin{array}{l}U \\
B \\
V \\
R \\
I\end{array}$ & $\begin{array}{c}- \\
- \\
3.99 \\
3.19 \\
2.88\end{array}$ & $\begin{array}{c}- \\
- \\
0.51 \\
0.44 \\
0.48\end{array}$ & $\begin{array}{c}- \\
- \\
55.3 \\
48.8 \\
45.1\end{array}$ & $\begin{array}{c}- \\
- \\
3.6 \\
3.9 \\
4.7\end{array}$ & 5 \\
\hline 13 & Vz145 & $\begin{array}{l}U \\
B \\
V \\
R \\
I\end{array}$ & $\begin{array}{l}5.48 \\
6.70 \\
6.29 \\
6.49 \\
5.55\end{array}$ & $\begin{array}{l}0.21 \\
0.07 \\
0.03 \\
0.04 \\
0.05\end{array}$ & $\begin{array}{l}62.6 \\
60.4 \\
60.7 \\
61.0 \\
60.4\end{array}$ & $\begin{array}{l}1.1 \\
0.3 \\
0.1 \\
0.2 \\
0.3\end{array}$ & 4 \\
\hline- & Vz14* & $\begin{array}{l}U \\
B \\
V \\
R \\
I\end{array}$ & $\begin{array}{c}- \\
2.04 \\
2.36 \\
2.45 \\
2.72\end{array}$ & $\begin{array}{c}- \\
0.32 \\
0.18 \\
0.17 \\
0.25\end{array}$ & $\begin{array}{c}- \\
59.7 \\
59.8 \\
55.9 \\
59.2\end{array}$ & $\begin{array}{c}- \\
4.7 \\
2.1 \\
2.0 \\
3.1\end{array}$ & 3 \\
\hline WR67 & Vz15 & $\begin{array}{l}U \\
B \\
V \\
R \\
I\end{array}$ & $\begin{array}{l}6.03 \\
6.88 \\
6.15 \\
6.42 \\
5.44\end{array}$ & $\begin{array}{l}0.32 \\
0.23 \\
0.07 \\
0.11 \\
0.12\end{array}$ & $\begin{array}{l}61.6 \\
60.0 \\
64.2 \\
65.5 \\
64.1\end{array}$ & $\begin{array}{l}1.5 \\
1.0 \\
0.3 \\
0.5 \\
0.6\end{array}$ & 6 \\
\hline- & Vz30* & $\begin{array}{l}U \\
B \\
V \\
R \\
I\end{array}$ & $\begin{array}{l}1.89 \\
1.50 \\
1.40 \\
1.70 \\
1.43\end{array}$ & $\begin{array}{l}0.36 \\
0.31 \\
0.18 \\
0.17 \\
0.09\end{array}$ & $\begin{array}{l}54.9 \\
49.3 \\
51.9 \\
58.3 \\
57.6\end{array}$ & $\begin{array}{l}5.2 \\
5.8 \\
3.7 \\
2.9 \\
1.7\end{array}$ & 4 \\
\hline- & Vz107* & $\begin{array}{c}U \\
B \\
V \\
R \\
I\end{array}$ & $\begin{array}{l}1.25 \\
1.62 \\
2.12 \\
2.03 \\
1.19\end{array}$ & $\begin{array}{c}0.41 \\
0.28 \\
0.14 \\
0.13 \\
0.27\end{array}$ & $\begin{array}{l}79.9 \\
60.9 \\
70.6 \\
72.2 \\
72.9\end{array}$ & $\begin{array}{l}8.8 \\
4.9 \\
1.9 \\
1.9 \\
6.5\end{array}$ & 1 \\
\hline- & Vz121 & $\begin{array}{l}U \\
B \\
V \\
R \\
I\end{array}$ & $\begin{array}{c}- \\
3.46 \\
4.49 \\
5.17 \\
4.37\end{array}$ & $\begin{array}{c}- \\
0.98 \\
0.21 \\
0.27 \\
0.36\end{array}$ & $\begin{array}{c}- \\
68.5 \\
62.9 \\
64.7 \\
66.3\end{array}$ & $\begin{array}{c}- \\
7.9 \\
1.3 \\
1.5 \\
2.3\end{array}$ & 3 \\
\hline- & Vz128 & $\begin{array}{l}U \\
B \\
V \\
R \\
I\end{array}$ & $\begin{array}{c}- \\
- \\
3.49 \\
4.46 \\
4.02\end{array}$ & $\begin{array}{c}- \\
- \\
0.22 \\
0.87 \\
0.17\end{array}$ & $\begin{array}{c}- \\
- \\
63.6 \\
66.6 \\
61.3\end{array}$ & $\begin{array}{c}- \\
- \\
1.8 \\
5.5 \\
1.2\end{array}$ & 3 \\
\hline- & Vz148 & $\begin{array}{c}U \\
B \\
V \\
R \\
I\end{array}$ & $\begin{array}{c}- \\
- \\
4.00 \\
5.29 \\
3.61\end{array}$ & $\begin{array}{c}- \\
- \\
0.23 \\
0.31 \\
0.44\end{array}$ & $\begin{array}{c}- \\
- \\
60.5 \\
60.3 \\
60.2\end{array}$ & $\begin{array}{c}- \\
- \\
1.7 \\
1.7 \\
3.5\end{array}$ & 10 \\
\hline
\end{tabular}

There is no previous spectroscopic information in the literature about stars 4, 10,12 and Vz148. For the rest of the objects
Table 1. continued.

\begin{tabular}{|c|c|c|c|c|c|c|c|}
\hline $\operatorname{Star}^{a}$ & Id. ${ }^{b}$ & Filter & $\begin{array}{l}P_{\lambda} \\
\%\end{array}$ & $\begin{array}{l}\epsilon_{\mathrm{p}} \\
\%\end{array}$ & $\begin{array}{c}\theta_{\lambda} \\
\circ\end{array}$ & $\begin{array}{c}\epsilon_{\theta} \\
\circ\end{array}$ & $n^{c}$ \\
\hline \multirow[t]{5}{*}{ - } & Vz182 & $U$ & 4.80 & 1.85 & 97.7 & 10.5 & 4 \\
\hline & & $B$ & 4.93 & 1.87 & 85.9 & 9.2 & \\
\hline & & $V$ & 5.67 & 0.39 & 56.1 & 2.0 & \\
\hline & & $R$ & 5.44 & 0.52 & 61.4 & 2.7 & \\
\hline & & $I$ & 4.89 & 1.43 & 55.6 & 8.2 & \\
\hline \multirow[t]{5}{*}{-} & Vz183* & $U$ & - & - & - & - & 1 \\
\hline & & $B$ & 1.74 & 0.37 & 47.1 & 6.0 & \\
\hline & & V & 1.97 & 0.21 & 60.3 & 3.1 & \\
\hline & & $R$ & 2.50 & 0.20 & 59.5 & 2.3 & \\
\hline & & $I$ & 1.52 & 0.44 & 61.4 & 8.1 & \\
\hline
\end{tabular}

${ }^{a}$ Lyngå (1986); * stands for nonmember.

${ }^{b}$ Vázquez et al. (1995); * stands for nonmember.

${ }^{c}$ Number of integrations.

Table 2. Polarization results of stars in Pismis 20.

\begin{tabular}{lcccccc}
\hline \hline Star $^{a}$ & $\begin{array}{c}P_{\max } \\
\%\end{array}$ & $\epsilon_{\mathrm{p}}$ & $\sigma_{1}^{b}$ & $\begin{array}{c}\lambda_{\max } \\
\mu \mathrm{m}\end{array}$ & $\epsilon_{\lambda}$ & Sp. T. \\
\hline 1 & 5.96 & 0.23 & 1.91 & 0.61 & 0.06 & - \\
2 & 6.18 & 0.51 & 1.78 & 0.55 & 0.09 & - \\
3 & 3.51 & 0.64 & 0.84 & 0.73 & 0.21 & B0 I-III \\
$4 *$ & 1.81 & 0.20 & 0.97 & 0.48 & 0.07 & - \\
7 & 3.98 & 0.32 & 1.45 & 0.59 & 0.07 & O9.5 Ib \\
8 & 6.17 & 0.12 & 1.23 & 0.57 & 0.02 & B2 Ia-O \\
9 & 4.80 & 0.29 & 1.11 & 0.55 & 0.04 & B0.2 III \\
10 & 6.95 & 0.28 & 2.14 & 0.58 & 0.05 & - \\
11 & 6.46 & 0.11 & 0.78 & 0.56 & 0.02 & O9.5Ib \\
12 & 3.91 & 0.37 & 0.52 & 0.48 & 0.05 & - \\
13 & 6.57 & 0.16 & 2.36 & 0.57 & 0.03 & O9 Vn \\
Vz14* & 2.58 & 0.13 & 1.13 & 0.67 & 0.07 & - \\
WR67 & 6.45 & 0.21 & 1.77 & 0.56 & 0.04 & WN6 + OB? \\
Vz30* & 1.68 & 0.13 & 1.30 & 0.57 & 0.06 & - \\
Vz107* & 1.99 & 0.17 & 1.50 & 0.56 & 0.09 & - \\
Vz121 & 4.88 & 0.18 & 1.02 & 0.69 & 0.05 & - \\
Vz128 & 4.06 & 0.07 & 0.51 & 0.76 & 0.03 & - \\
Vz148 & 4.52 & 0.65 & 2.08 & 0.70 & 0.21 & - \\
Vz182 & 5.71 & 0.04 & 0.63 & 0.56 & 0.01 & - \\
Vz183* & 2.23 & 0.25 & 1.42 & 0.69 & 0.14 & - \\
\hline
\end{tabular}

${ }^{a}$ Identifications from Lyngå (1968) or from Vázquez et al. (1995; Vz). * Stands for nonmember.

${ }^{b} \sigma_{1}^{2}=\sum\left(r_{\lambda} / \epsilon_{\mathrm{p}_{\lambda}}\right)^{2} /(m-2)$; where $m$ is the number of colors and $r_{\lambda}=P_{\lambda}-P_{\max } \exp \left(-K \ln ^{2}\left(\lambda_{\max } / \lambda\right)\right)$.

with indications of a non-interstellar origin in their polarizations, VZ95 suggest that No. 2 might have a close unresolved companion, in which case the detected intrinsic polarization could be due to dust associated with the binary system. The same explanation seems to be valid for star 13. It has been classified as O9.5 Vn, Bassino et al. (1982) and Papadópulos (1985) reported changes in its radial velocity, which could be explained assuming the star as a binary. Numero 7 is a $09.5 \mathrm{Ib}$ 

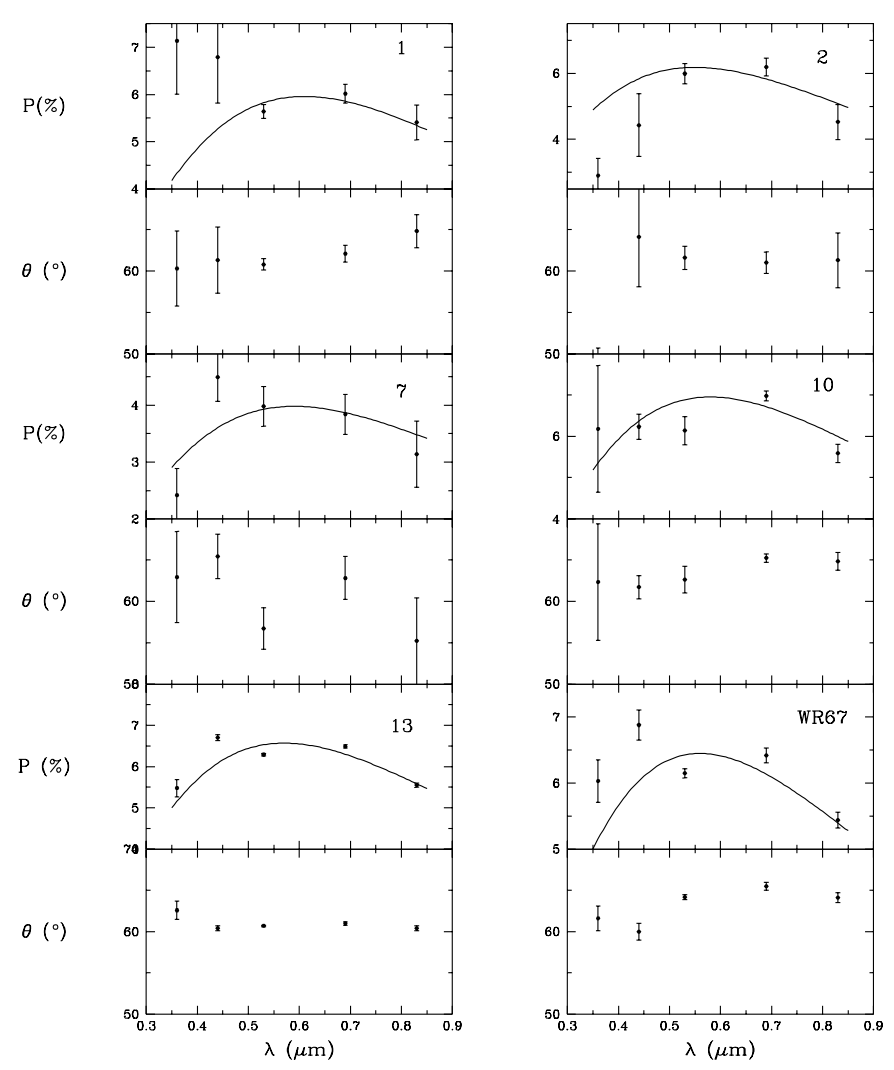

Fig. 1. Polarization and position angle dependence on wavelength for stars with indications of intrinsic polarization (mismatch between observations and Serkowski's curve fit and/or variable position angle).

object (Papadópulos 1985); and finally, WR 67 shows a high polarization value $(6.45 \%)$ which in part may be intrinsic.

It is known that for the interstellar medium the polarization efficiency (ratio of the maximum amount of polarization to visual extinction) cannot exceed the empirical upper limit

$P_{\text {max }} \leq 3 A_{\mathrm{v}} \simeq 3 R_{\mathrm{v}} E_{B-V}$

obtained for interstellar dust particles (Hiltner 1956). The ratio $P_{\max } / E_{B-V}$ depends mainly on the alignment efficiency and the magnetic field strength, and also on the amount of depolarization due to radiation traversing more than one cloud with different field directions. Figure 2 depicts the relation that exists between the reddening and the polarization originating in the dust along the line of sight to Pismis 20. The empirical upper limit (2), known as the line of maximum efficiency, has been drawn adopting $R=3.2$. Excesses $E_{B-V}$ were obtained from the literature or from the relation between spectral type and color indices following Schmidt-Kaler (1982). For WR 67 the values used are carefully explained in the next section. As can be seen, the objects lie to the right of the interstellar maximum line, a situation that indicates that the observed polarization is mostly due to the interstellar material.

This figure also shows that the observed stars segregate into three groups. There is a certain number of stars in the lower part of the plot (Nos. 4, 14, Vz30, Vz107 and Vz183; filled triangles) that were previously identified as nonmembers by LY68 or by VZ95. This group has a polarization which amounts to $\overline{P_{\max }}=2.06 \pm 0.5 \%$ (vectorial mean) and $\overline{\theta_{\mathrm{v}}}=58.6$.

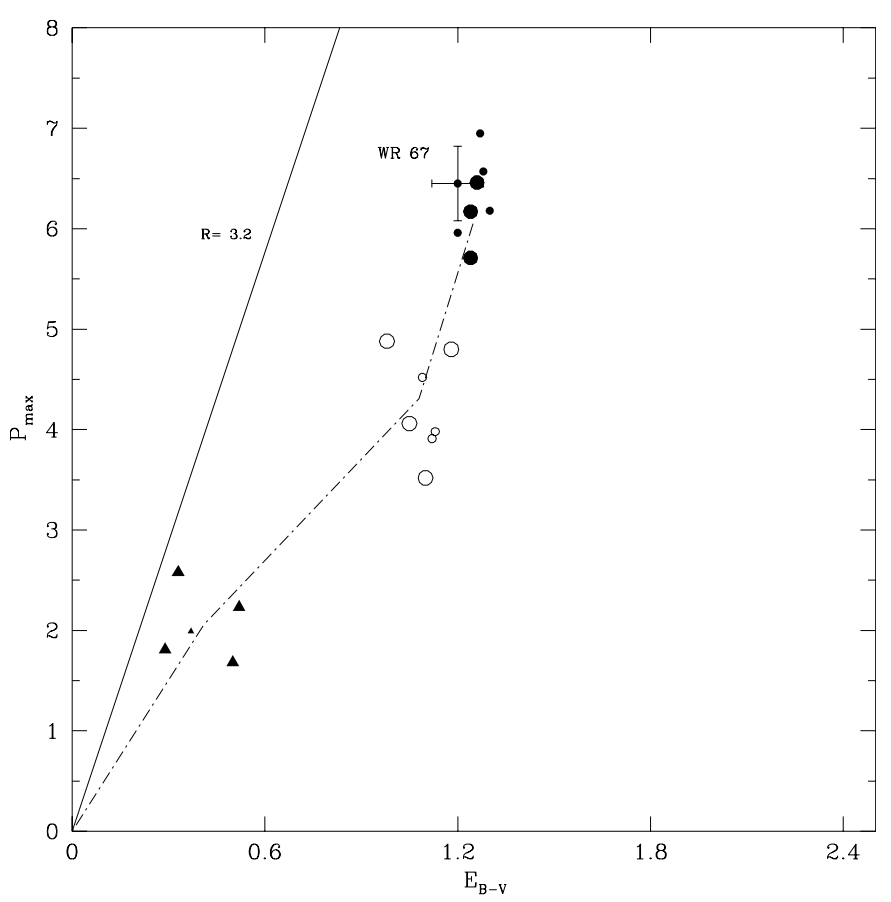

Fig. 2. Polarization efficiency diagram for the observed parameters. Triangles are used for foreground stars, open circles for stars in group A and filled circles for stars in group B. Small symbols are used for stars with indications of intrinsic polarization. The dash-dotted line shows the change in dust efficiency between groups. See the text for the explanation of the error bars drawn only for WR 67.

It originates in a nearby dust cloud which Neckel \& Klare (1980, Fig. 9) locate in the Local arm at a distance of less than $1 \mathrm{kpc}$ from the Sun in direction to Pismis 20. Behind these stars lies a second sheet of absorption, also shown by them to be at about $1.2 \mathrm{kpc}$. The rest of the stars clearly split into two groups, which we have identified with the names A and B (open and filled circles, respectively). Group A, comprising 7 objects (stars Nos. 3, 7, 9, 12, Vz121, Vz128, Vz148), has a $\overline{P_{\max }}=4.31 \pm 0.5 \%$ (vectorial mean of 4 stars, not including those with intrinsic polarization), $\overline{\theta_{\mathrm{v}}}=63.5$ (same restrictions) in equatorial coordinates ( $94^{\circ} 6$ in Galactic coordinates) and $\overline{E_{B-V}}=1.08$ mag. Group B has 8 members (stars 1, 2, 8, 10, $11,13, \mathrm{Vz} 182$ and, possibly, WR 67) with $\overline{P_{\max }}=6.08 \pm 0.8 \%$ (mean of 3 stars, same restrictions), $\overline{\theta_{\mathrm{v}}}=62^{\circ} .6$ in equatorial coordinates (93.7 in Galactic coordinates) and a $\overline{E_{B-V}}=1.25$ mag. Note that the existence of two separate groupings within cluster stars is based only on the existence of a gap between $P_{\max }=4.9 \%$ and $5.7 \%$ among stars with observational errors $\epsilon_{\mathrm{p}} \simeq .25 \%$.

The plot of individual Stokes parameters of the polarization vector $(Q=P \cos 2 \theta$ and $U=P \sin 2 \theta)$ for each of the observed stars (Fig. 3) shows again these three groupings. The dash-dotted line represents the changing direction of the vector $\boldsymbol{P}$ while connecting the mean $(Q, U)$ values for the 3 different groups. A slight change in direction of the $\boldsymbol{P}$ vector can be seen between groups A and B, which indicates different polarimetric characteristics in the dust causing the polarization. The wavelength for which the maximum polarization is observed $\left(\lambda_{\max }\right)$ is related to the mean size 


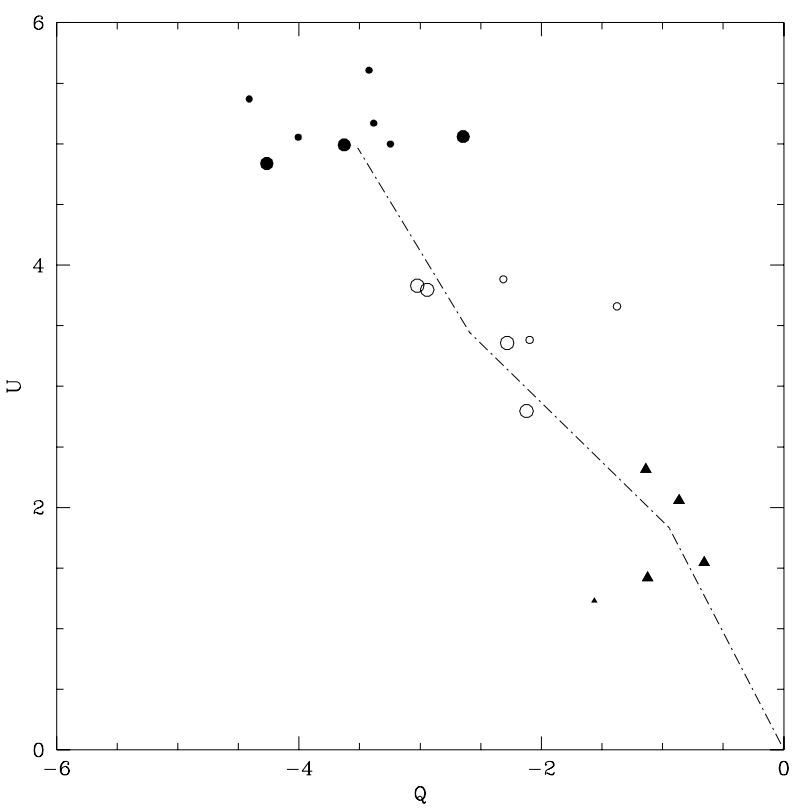

Fig. 3. Plot of individual Stokes parameters $Q$ and $U$ for the observed stars. Symbols are as indicated in Fig. 2. The dash-dotted line shows the change in direction of the polarization vector between groups.

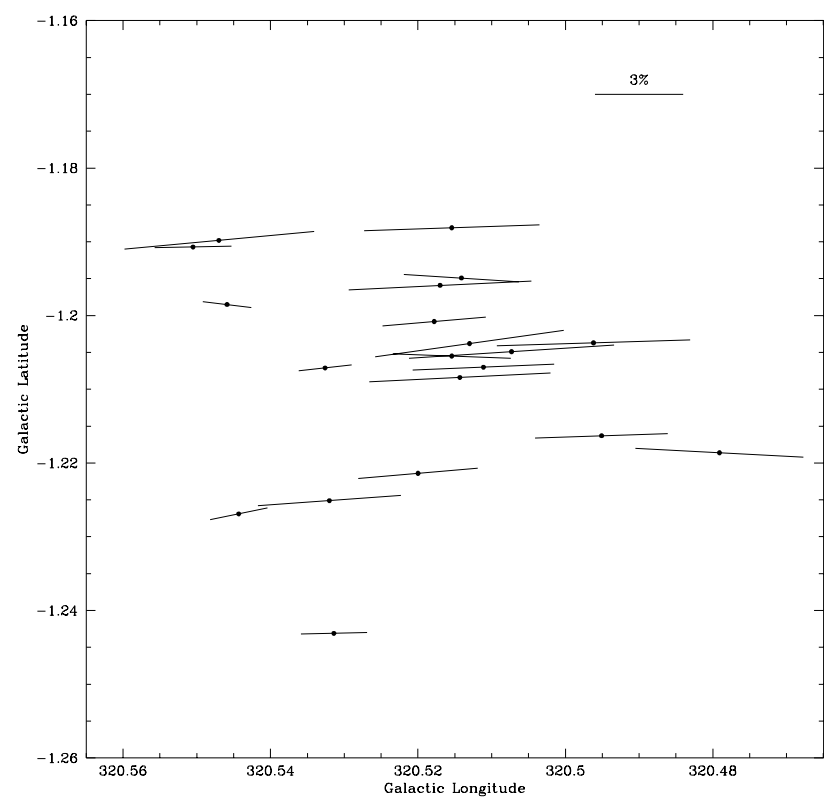

Fig. 4. Observed polarization vectors and their orientations for the observed stars in the direction of Pismis 20. The length of each vector is proportional to the percentage polarization.

of the particles. Through their values for groups A and B, $\lambda_{\max _{\mathrm{A}}}=0.66 \pm 0.08 \mu \mathrm{m}$ and $\lambda_{\max _{\mathrm{B}}}=0.57 \pm 0.02 \mu \mathrm{m}$, we conclude that in both cases we are dealing with particles with a size close to the normal value $(0.55 \mu \mathrm{m})$ within the errors. Figure 4 plots polarization vectors and their orientations for the observed stars in the direction of the cluster. Figure 5 shows a similar plot but for stars in the neighborhood of the open cluster, with information from the catalogue of Heiles (2000). The alignment of the e-vectors along the galactic plane dominates both field stars (at a wide range of distances) and cluster stars (supposedly at a fixed distance).

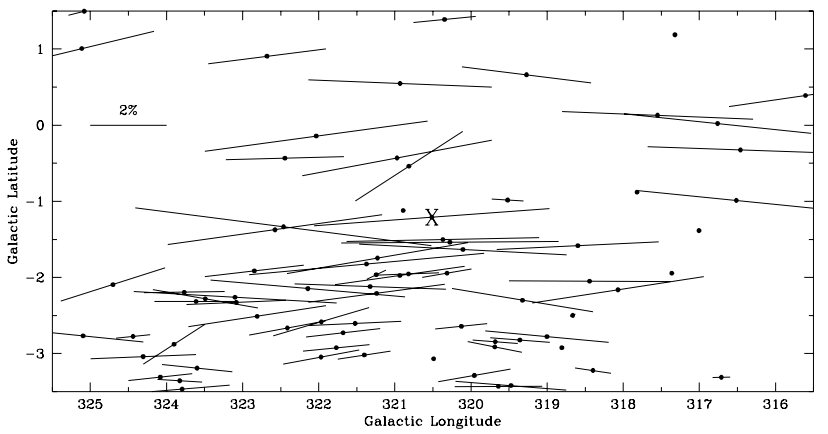

Fig. 5. Polarization vectors and their orientations for stars from Heiles (2000) in the neighborhood of Pismis 20. The length of each vector is proportional to the percentage polarization. The approximate position of the cluster is indicated with a cross.

\section{Conclusions}

The high measured polarizations in the direction of Pismis 20 results from the contribution of several dust layers. There exists a dust cloud at less than $1 \mathrm{kpc}$ from the Sun, in the Local arm, which is responsible for only part of the measured polarizations in the direction of the cluster. The rest originates in other two dust clouds, one of them situated in front of the cluster and another one located between the two groups, A and B.

Both groups (A and $\mathrm{B}$ ) clearly differ in their polarimetric characteristics: mean polarization, polarization efficiency and, to a lesser degree, direction of the e-vector. There are two possible explanations for our findings. The first one is that polarizing dust particles exist between certain members of the cluster, with a different chemical composition in comparison with the foreground dust, responsible for the difference in mean polarization $(1.77 \%)$. In fact, the existence of varying amounts of matter inside the cluster has been suggested by Sagar et al. (2001). The polarization efficiency of the dust between A and B is $22 \%$ higher than the corresponding value for the particles that polarize the light from the stars in A.

The other explanation would be that Pismis 20 is composed of two groups superimposed along the line of sight. In this case, group B is a loose clustering located farther from the Sun than group A and the difference found in mean polarization of both groups would be due to dust between both locations. Group B is composed of some very luminous and evolved stars while in Group A there are some less luminous objects and it may include most of the faint objects found by VZ95.

Both groups cannot be separated in terms of distance since the observed part of the main sequence is too steep to obtain reliable determinations. Thus, even though we discern the existence of two apparently different groups within the stars previously considered members of this cluster, we cannot decide between neither explanation about the relative locations of the groups.

We have to mention the high percentage of stars in group B with indications of a non-interstellar origin in their polarizations $(60 \%)$ while for A this percentage drops to $20 \%$. Possible explanations are unresolved binarity, a consequence of their evolutive status and also the possibility of some undetected existence of gas. 
In order to relate the Wolf-Rayet star WR 67 with any of the groups we must analyze carefully its interstellar parameters: color excess and polarization. Wolf-Rayet stars are known to exhibit intrinsic values for both parameters. The interstellar color excess as measured by the $E_{B-V}$ is taken from Table 24 of van der Hucht (2001) as $1.20 \pm 0.08 \mathrm{mag}$. It should be remembered that some of these determinations are based solely upon the membership of WR 67 in the cluster Pismis 20, and hence are obtained by looking at the interpolated apparent interstellar color excess at the position of the WR star within the patchy cluster absortion; see for instance the discussion in Turner (1996). On the other hand, regarding polarizations, Harries et al. (1998) have shown that the intrinsic values of WR polarizations rarely exceed $0.3 \%$. Also, our observations were obtained in 6 integrations during the 5 observing nights over two seasons one year apart. This means that the polarization is not variable within the errors quoted in Table 1 . At the same time the $\sigma_{1}$ value (Table 2) for WR 67 can be interpreted in the sense: "departures from interstellar polarization times internal rms errors". Using this last approach we can put upper limits on the range of possible values of the intrinsic polarization of this star by multiplying $\sigma_{1} \times \epsilon_{\mathrm{p}}$ obtaining for the interstellar polarization $6.45 \% \pm(0.21 \% \times 1.77)=6.45 \% \pm 0.37 \%$. The conclusion is that the Wolf-Rayet WR 67 has a polarization value which relates to group $\mathrm{B}$ whilst its color excess is not informative in this matter.

Hron et al. (1985) have measured barycentric radial velocities of three stars in Pismis 20. They are: star No. 7 (-67.2 v? $\mathrm{km} \mathrm{s}^{-1}$; group A), star No. 8 (-71.9 v? $\mathrm{km} \mathrm{s}^{-1}$; group B) and No. $11\left(-10.2 \mathrm{~km} \mathrm{~s}^{-1}\right.$; group B). Due to the variability present in the first two stars, nothing can be said at this moment about the existence of radial velocity differences between the groups. The third star, No. 11, is recognized as a member of Pismis 20 in all the studies on the cluster on the basis of its photoelectric magnitudes and colors. For that, the listed radial velocity (based on one spectrogram) could indicate that it is possibly part of a binary system. More interesting are the radial velocities of the interstellar Ca II lines listed in the same article for stars Nos. 7 and $8:-25 \mathrm{~km} \mathrm{~s}^{-1}$ and $-67.3 \mathrm{~km} \mathrm{~s}^{-1}$ respectively. They point to a difference in the interstellar material between the spatial location of those stars, which do not belong to the same group, well beyond measurement errors. This is in line with our polarimetric observations.

We think that a deep study of the cluster Pismis 20 through stellar radial velocities and also of the interstellar Ca II lines could be of value. If A and B are subgroups in Pismis 20, the stellar radial velocities may not be decisive in confirming this situation, but if we were dealing with two different clusters superposed along the line of sight, radial velocity measurements would show it. Also, more measurements of the interstellar lines would help to confirm the presence of interstellar material between both groups.

Acknowledgements. We wish to acknowledge Dr. Nidia Morrell for helpful discussions and the technical support and hospitality at CASLEO during the observing runs. We also acknowledge the use of the Torino Photopolarimeter built at Osservatorio Astronomico di Torino (Italy) and operated under agreement between Complejo Astronomico El Leoncito and Osservatorio Astronomico di Torino. Special thanks go to Carlos Feinstein for valuable comments, and to Mrs. M. C. Fanjul de Correbo and Mr. Ruben Martínez for technical assistance.

\section{References}

Bassino, L. P., Dessaunet, V. H., Muzzio, J. C., \& Waldhausen, S. 1982, MNRAS, 201, 885

Clocchiati, A., \& Marraco, H. G. 1988, A\&A, 197, L1

Harries, T. J., Hillier, D. J., \& Howarth, I. D. 1998, MNRAS, 296, 1072

Heiles, C. 2000, AJ, 119, 923

Hiltner, W. A. 1956, ApJS, 2, 389

Hron, J., Maitzen, H. M., Moffat, A. F. J., Schmidt-Kaler, Th., \& Vogt, N. 1985, A\&AS, 60, 355

Lortet, M. C., Georgelin, Y. P., \& Georgelin, Y. M. 1987, ApJS, 35, 111

Lyngå, G. 1968, The Observatory, 88, 20 (LY68)

Lundström, I., \& Stenholm, B. 1984, A\&A, 58, 163

Maeder, A., \& Meynet, G. 1988, A\&AS, 76, 411

Massey, M. P., DeGioia-Eastwood, K., \& Waterhouse, E. 2001, AJ, 121,1050

McMillan, R. S. 1978, ApJ, 225, 880

Moffat, A. F. J., \& Vogt, N. 1973, A\&AS, 10, 135

Neckel, T. H., \& Klare, G. 1980, A\&AS, 42, 251

Papadópulos, S. 1985, Thesis, unpublished, IAFE, Univ. of Buenos Aires, Argentina

Paunzen, E., Pintado, O. I., \& Maitzen, H. M. 2002, A\&A, 395, 823

Peterson, C. J., \& FitzGerald, M. P. 1988, MNRAS, 235, 1439

Pismis, P. 1959, Bol. Obs. Tonantzintla Tacubaya, 18, 37

Sagar, R., Munari, U., \& de Boer, K. S. 2001, MNRAS, 327, 23

Schmidt-Kaler, Th. 1982, in Landolt/Bornstein, Neue Series VI/2b

Serkoswki, K. 1973, in Interstellar Dust and Related Topics, ed. J. M. Greenberg, \& H. C. van der Hulst (Dordrecht-Holland: Reidel), IAU Symp., 52, 145

Serkowski, K., Mathewson, D. L., \& Ford, V. L. 1975, ApJ, 196, 261

Turner, E. G. 1996, AJ, 111, 828

van der Hucht, K. A. 2001, NewAR, 45, 135

Vázquez, R. A., Will, J. M., Prado, P., \& Feinstein, A. 1995, A\&AS, 111, 85 (VZ95)

Wilking, B. A., Lebofsky, M. J., Martin, P. G., Rieke, G. H., \& Kemp, J. C. 1980, ApJ, 235, 905 